\title{
Group fitness is effective in reducing cardiovascular disease risk factors in healthy adults
}

\author{
Jinger S. Gottschall ${ }^{1 \#}$, Justin L. Jones ${ }^{1}$, Jackie Mills ${ }^{2}$, Bryce Hastings ${ }^{2}$ \\ ${ }^{1}$ The Pennsylvania State University, University Park, USA; ${ }^{\#}$ Corresponding Author: jinger@psu.edu \\ ${ }^{2}$ Les Mills International, Auckland City, New Zealand
}

Received 18 November 2012; revised 20 December 2012; accepted 27 December 2012

\section{ABSTRACT}

In the past 5 years, 1 of every 3 deaths in the United States was attributed to cardiovascular disease (CVD). Regular physical activity has numerous benefits associated with the reduction of cardiovascular disease risk factors. Despite these details, $60 \%$ of Americans are not regularly physically active and $25 \%$ are not active at all. Previous studies have found significant changes in cardiovascular disease risk factors by combining cardiorespiratory and strength training into one exercise program. The current pilot study augments the approach by utilizing a group fitness program that mimics the Physical Activity Guidelines for Fitness. Therefore our purpose was to evaluate if a multimodal group fitness intervention could singularly produce the physiological and musculoskeletal health benefits of the established physical activity prescription. Twenty-five sedentary, but otherwise healthy, adults (15 women and 10 men) between the ages of 25 - 40 years completed the protocol of a 30-week group exercise program. It started with a 6-week familiarization period, continued with a 12-week block of 6 group fitness classes per week ( 3 cardiovascular, 2 strength, 1 flexibility), and concluded with a 12-week block of 7 classes per week (4 cardiovascular, 2 strength, 1 flexibility). We completed submaximal oxygen consumption treadmill tests, fasting blood draws and iDXA scans at 3 time points (baseline, midpoint, final) and compared the data using a paired t-test $(p<0.05)$. Compared to baseline measurements, the final measurements demonstrated that the participants had statistically

\footnotetext{
*Conflict of interest: While Les Mills International was supportive of the present study they did not fund the intervention nor did they have access to the data for analyses.
}

significant reductions in body mass, fat body mass percentage, total cholesterol, LDL-C, and triglycerides and elevations in oxygen consumption, lean body mass percentages, and HDL-C. Based upon our results, group fitness may be an ideal method to minimize attrition and maximize health benefits in a comprehensive manner to reduce the risk of cardiovascular disease.

Keywords: Physical Fitness; Physical Activity; Exercise Intervention

\section{INTRODUCTION}

In the past 5 years, 1 of every 3 deaths in the United States was attributed to cardiovascular disease (CVD), which equates to 2200 Americans each day or 1 death every 30 seconds $[1,2]$. A sedentary lifestyle is one of two primary behavioral factors associated with the advancement of CVD. Despite these alarming statistics, $60 \%$ are not regularly physically active and $25 \%$ are not active at all [3]. It is critical that research scientists, medical practitioners, and funding agencies strive to obtain easy to follow exercise prescriptions to reverse these trends. Regular physical activity has numerous benefits associated with decreasing the risk of cardiovascular disease such as the reduction of total body fat mass, low density lipoprotein cholesterol (LDL-C), triglycerides, blood pressure, and elevation of maximal oxygen consumption, high density lipoprotein cholesterol (HDL-C) and glucose tolerance [4].

The American College of Sports Medicine (ACSM) and the United States Department of Health and Human Services established the Physical Activity Guidelines for Fitness [1] to provide direction for healthy individuals. They suggest that adults engage in cardiovascular exercise of moderate-intensity for $30-60$ minutes at least 5 days per week, or vigorous-intensity for 20 - 60 minutes 
at least 3 days per week, or a combination of these moderate- and vigorous-intensity regimens 3 - 5 days per week. In addition, the position stand recommends that adults engage in a full body muscular strength routine 2 days per week as well as flexibility exercises $1-2$ days per week.

Past research demonstrates that implementing an exercise program with both cardiovascular activities and strength training, without any dietary changes, can reduce cardiovascular disease risk factors. Park et al. [5] compared the data from a group of participants who completed a 24-week combination cardiorespiratory and strength training protocol to both a cardiorespiratory training only protocol and a control group. The combined training program was the most effective in reducing CVD risk factors as mean body fat percentage decreased by $11.2 \%$, LDL-C decreased by $34.7 \%$, and triglycerides decreased by $42.3 \%$ from baseline. In addition, HDL-C increased by $23.1 \%$ and lean body mass increased by $10.3 \%$. In a similar study, Libardi et al. [6] studied inactive participants who completed concurrent cardiorespiratory and strength training for 16 weeks in comparison to three other study groups; resistance training only, cardiorespiratory training only, and a control group. Similar to previous reports, the combined protocol yielded the most significant results whereby total cholesterol concentration decreased by $27.8 \%$ and triglycerides decreased by $33.4 \%$. McCarthy et al. [7] also evaluated the effects of a concurrent training program on sedentary men for a 10-week training program of cardiorespiratory and full body strength training. Total body weight did not statistically decrease, however fat percentage decreased from $19.5 \%$ to $17.2 \%$.

These studies found significant changes in cardiovascular disease risk factors by combining cardiorespiratory and strength training into one exercise program. The current study augments the approach by utilizing a group fitness program that mimics the Physical Activity Guidelines for Fitness. A Nielsen survey of over 3000 participants in group fitness classes across the globe showed that more than $85 \%$ of these members visit their clubs at least 2 days per week specifically to engage in classes and nearly $43 \%$ of these members visit their club 4 times a week for this purpose [8]. To add, the International Health, Racquet and Sportsclub Association (IHRSA) estimates that United States club members attend their gyms 3.4 times a week to attend group fitness classes [9]. Thus, group fitness may be one method to minimize attrition and thereby maximize health benefits in a comprehensive and holistic manner. We hypothesize that a 30-week multimodal group fitness intervention will produce beneficial effects on CVD risk factors by reducing body weight, total cholesterol, LDL-C, triglycerides concentration, systolic blood pressure, diastolic blood pressure, and by enhancing lean body mass percentage, HDL-C, glucose tolerance and maximal oxygen consumption.

\section{METHODS}

Twenty-nine healthy adults, 18 women and 11 men, started the program and 25 participants, 15 women and 10 men (Figure 1, Table 1) finished the program. All of the participants gave written informed consent that followed the guidelines of The Pennsylvania State University Human Research Committee. The participants were physically inactive, but otherwise healthy, and completed a health exam at the beginning of the study to determine if physical activity was appropriate. The initial inclusion criteria for the study was for the participant to be between the age of 25 and 40, completing less than 30 minutes of exercise per week for the previous 6 months, and available from May to December without more than four days of travel in a single week. We excluded potential participants from the study, in accordance to the ACSM health screening for physical activity, if he or she had any of the following conditions: chest discomfort with exertion, unreasonable shortness of breath, symptoms of dizziness, fainting or blackouts, heart medication, asthma or other lung disease, burning or cramping sensations in lower legs with minimal physical activity, joint problems that limit physical activity, prescription medications, pregnancy, diabetes, smoking, blood pressure greater than $140 / 90 \mathrm{mmHg}$, or total blood cholesterol greater than $220 \mathrm{mg} \cdot \mathrm{dL}^{-1}$.

\subsection{Laboratory Measurements}

We collected musculoskeletal and physiological data at baseline (Week 3), midpoint (Week 19) and final (Week 31) time points of the study. The majority of the measures were collected at the university Clinical Research Center such as height, weight, blood pressure, blood concentrations and body composition variables. Blood was drawn from the antecubital vein after a 48-hour alcohol and a 12-hour food fast. The samples were assayed (Quest Diagnostics, Pittsburgh, PA) with a 24-Chem analysis, which included total cholesterol, LDL-C, HDL-C, triglycerides, and glucose. Body composition measurements were collected using a total-body scanner (GE Lunar iDXA, Madison, WI). Scan analysis was performed using GE Encore 11.10 software. This software allows for adjustment of regions of interest including the sagittal line, which controls the left-right body distribution of tissue.

Immediately following this research center visit, we conducted a submaximal oxygen consumption test on a treadmill at the Biomechanics Laboratory. We measured the rates of oxygen consumption (VO2) using an open 


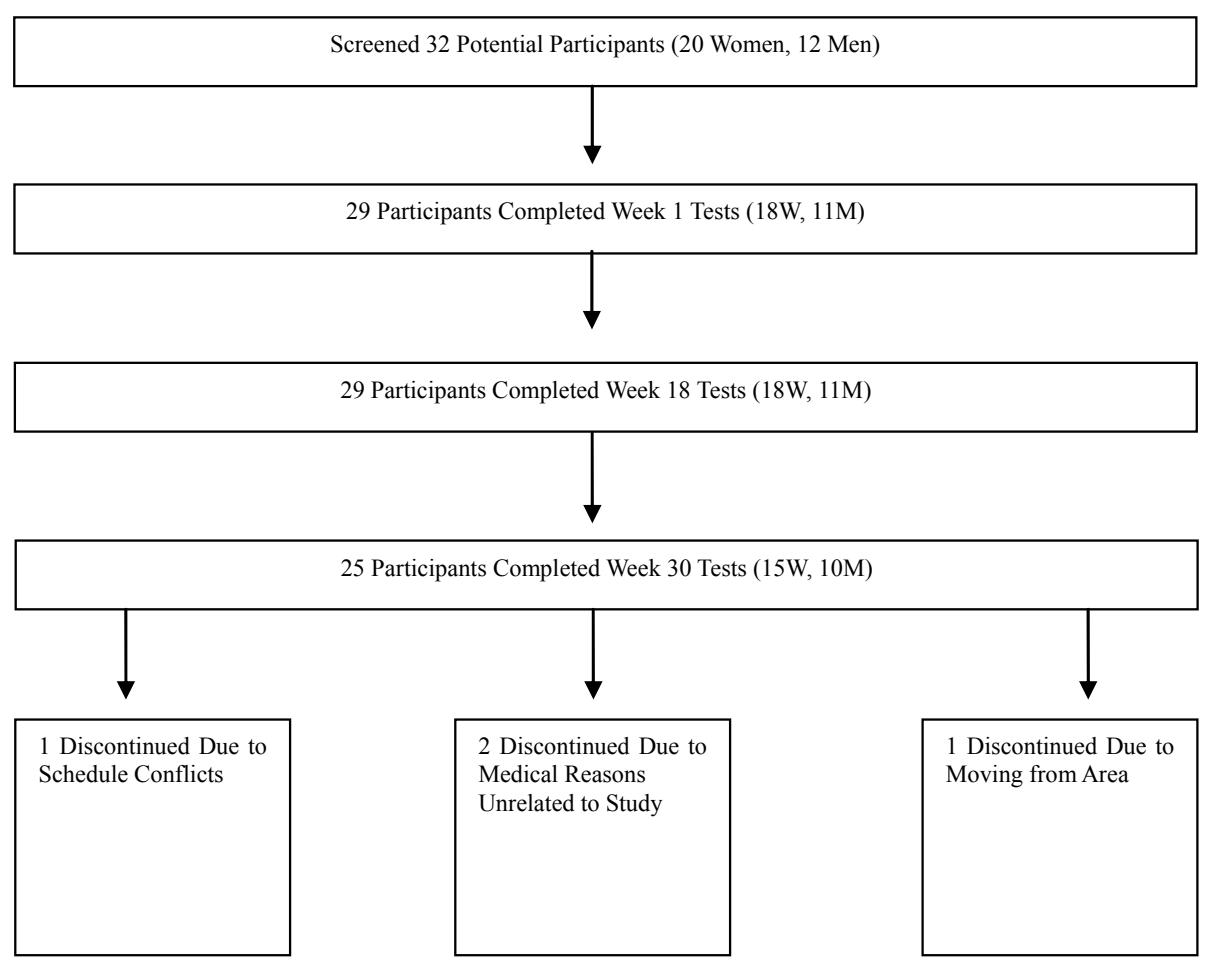

Figure 1. Flow chart designating the number of participants that completed each stage of the exercise intervention as well as the reasons for discontinuing.

Table 1. Participant $(n=25)$ characteristics at initial measurement; mean (standard deviation).

\begin{tabular}{lcc}
\hline & Men & Women \\
\hline Age (yr) & $32.3(2.4)$ & $30.4(4.5)$ \\
Height (cm) & $177.8(5.2)$ & $163.0(6.4)$ \\
BMI $\left(\mathbf{k g} \cdot \mathbf{m}^{-2}\right)$ & $29.0(5.5)$ & $30.0(5.5)$ \\
Number Overweight & 1 & 6 \\
Number Obese & 4 & 7 \\
\hline
\end{tabular}

circuit respirometry system (ParvoMedics, Sandy, UT). For the submaximal treadmill protocol we utilized workload and heart rate data from two submaximal stages of a treadmill walking test. Treadmill speed was 3.4 miles per hour throughout the submaximal test after a standing trial. First we conducted an economy test; the participants walked for 10 minutes on the level treadmill. Next we increased the grade by $3 \%$ every 3 minutes for women and $4 \%$ every 3 minutes for men. We recorded heart rate every 5 seconds and concluded the test when participant's heart rate was above 140 for 15 seconds. Finally, we performed a recovery test; the participants walked for 2 minutes at 2.5 miles per hour and 2 minutes at 2.0 miles per hour. We estimated VO2 max with the following equations.

$$
\text { slope: }(\mathrm{b})=(\mathrm{SM} 2-\mathrm{SM} 1) /(\mathrm{HR} 2-\mathrm{HR} 1)
$$

SM1 and SM2 are the average submaximal oxygen consumption values at 2 of the 3-minute stages of steady state. HR1 and HR2 are the average heart rate values at the same stages as the oxygen consumption values. maximal oxygen consumption estimation:

$$
\mathrm{VO} 2=\mathrm{SM} 2+\mathrm{b}(\mathrm{HR} \max -\mathrm{HR} 2)
$$

\subsection{Physical Fitness Program}

The group fitness program began with a 6-week introduction block where we gradually introduced the participants to the fitness classes and progressively increased their amount of exercise time in an effort to reduce injury and optimize retention (Table 2). After the 6-week familiarization period the participants completed 2,12 -week blocks of $6-7$ classes per week. The difference between the 2,12-week exercise blocks was that the first block included 3 cardiorespiratory classes and the second block included 4 cardiorespiratory classes (Table 2).

The participants completed Les Mills ${ }^{\mathrm{TM}}$ instructed group fitness classes at The North Club in State College, Pennsylvania. Each class was offered nearly every day of the week with classes beginning at 5:45 am until $6: 45 \mathrm{pm}$ allowing each participant to create an individual exercise schedule on a weekly basis. The prescribed fitness classes were the only form of physical activity, aside from daily living, for the participants during the 
Table 2. Group fitness protocol.

\begin{tabular}{clll}
\hline Week & Cardiorespiratory & Muscular Strength & Flexibility \\
\hline 1 & 20 minutes $(1 \times 20)$ & 20 minutes $(1 \times 20)$ & 10 minutes $(1 \times 10)$ \\
2 & 40 minutes $(2 \times 20)$ & 30 minutes $(1 \times 30)$ & 20 minutes $(1 \times 20)$ \\
3 & 60 minutes $(3 \times 20)$ & 45 minutes $(1 \times 30,1 \times 15)$ & 30 minutes $(1 \times 30)$ \\
4 & 90 minutes $(3 \times 30)$ & 60 minutes $(1 \times 60)$ & 40 minutes $(1 \times 40)$ \\
5 & 120 minutes $(3 \times 40)$ & 80 minutes $(1 \times 60,1 \times 20)$ & 50 minutes $(1 \times 50)$ \\
6 & 150 minutes $(3 \times 50)$ & 100 minutes $(1 \times 60,1 \times 40)$ & 60 minutes $(1 \times 60)$ \\
$7-18$ & 180 minutes $(3 \times 60)$ & 120 minutes $(2 \times 60)$ & 60 minutes $(1 \times 60)$ \\
$19-30$ & 240 minutes $(4 \times 60)$ & 120 minutes $(2 \times 60)$ & 60 minutes $(1 \times 60)$ \\
\hline
\end{tabular}

intervention. The participants independently selected their cardiorespiratory classes on a weekly basis from BodyAttack $^{\text {TM }}$ (sports-inspired intervals), BodyCombat ${ }^{\text {TM }}$ (mixed martial arts), BodyStep ${ }^{\mathrm{TM}}$ (height-adjust-able bench) and $\mathrm{RPM}^{\mathrm{TM}}$ (indoor cycling). The muscular strength class, BodyPump $^{\mathrm{TM}}$ incorporates a barbell as well as free weights. It challenges all the major muscle groups by using classic exercises such as, squats, lunges, chest and triceps presses, dead lifts and biceps curls. Finally, the flexibility class, BodyBalance ${ }^{\mathrm{TM}} /$ Body-Flow $^{\mathrm{TM}}$ is a yoga, tai chi, and pilates fusion workout. It is a carefully structured series of stretches and poses for the improvement and maintenance of functional mobility.

All data were analyzed between baseline values and the final values using paired t-tests with significance being defined as $\mathrm{p}<0.05$.

\section{RESULTS}

In support of our hypotheses, the 30-week group fitness class program resulted in statistically significant reductions in body mass, fat body mass percentage, total cholesterol, LDL-C, and triglycerides and elevations in oxygen consumption, lean body mass percentages, and HDL-C (Table 3, Figures 2-4). However, there were no significant differences in either systolic or diastolic blood or fasting glucose tolerance (Figures 5 and 6). To add, the compliance rate was $98.8 \%$, with 20 of the 25 participants never missing a prescribed session.

Body composition differed between initial and final measurements in both men and women in terms of mass as well as percent fat and lean tissue. Mean body mass of the women (Table 3) decreased by 3.8\% from the baseline body mass $(\mathrm{p}<0.001)$ with a $4.1 \%$ decrease in body fat percentage $(\mathrm{p}<0.001)$ while mean Body Mass Index (BMI) decreased from $30.0 \mathrm{~kg} \cdot \mathrm{m}^{-2}$ to $28.9 \mathrm{~kg} \cdot \mathrm{m}^{-2}$. Similarly, mean body mass of the men decreased by $5.1 \%$ (Table 3, p $=0.001$ ), while the mean BMI decreased from $29.0 \mathrm{~kg} \cdot \mathrm{m}^{-2}$ to $27.2 \mathrm{~kg} \cdot \mathrm{m}^{-2}$. On an individual basis, five of the fifteen women and seven of the ten men had at least a $5.0 \%$ decrease in body mass. In addition, the final measurement for lean tissue percentage was a mean $4.1 \%$ greater for the women and $6.9 \%$ greater for the men compared to the initial measurement.

There were also numerous statistically significant differences between the initial and final measurements of the blood profile in both women and men. As an example, the mean total cholesterol, as a combination of the women and men, decreased by $6.2 \%(\mathrm{p}<0.05$, Figure 2(a)). For the woman only, LDL-C decreased by $7.2 \%$ (p $<0.05$, Figure 2(b)) whereas, HDL-C concentration and triglycerides did not differ between measurements. For the men only, LDL-C decreased by $13.2 \%$, HDL-C increased by $12.2 \%$, and triglycerides decreased by $29.9 \%$ (all values, $\mathrm{p}<0.01$, Figures 2 and $\mathbf{3}$ ). There was no significant difference in fasting glucose, systolic blood pressure or diastolic pressure in either the men or the women (Figures 5 and $\mathbf{6}$ ).

Oxygen consumption significantly increased in both women and men during the 30-week group fitness intervention (Figure 4). For the women, cardiovascular fitness improved by $49.6 \%$, from 29.1 to $43.5 \mathrm{ml} / \mathrm{kg} / \mathrm{min}$ and for the men, fitness improved by $63.5 \%$ from 31.6 to $51.7 \mathrm{ml} / \mathrm{kg} / \mathrm{min}$.

\section{DISCUSSION}

Based upon our results, group fitness may be an ideal method to minimize attrition and maximize health benefits in a comprehensive manner. The 30 -week intervention was effective in reducing multiple cardiovascular disease risk factors such as high body mass and fat percent, reducing total cholesterol, LDL-C and triglycerides, and enhancing HDL-D as well as oxygen consumption.

The body mass and percent fat data reduce a primary cardiovascular disease risk factor of overweight and obesity. The ACSM states that a $2 \%$ to $5 \%$ reduction in body mass can lead to reductions in health risks and add that clinically significant reduction in body mass is defined by having a $5 \%$ change [10]. Additionally, the Strategies to Overcome \& Prevent (STOP) Obesity Alliance, a collaborative group of government, labor, business, health 
Table 3. Body composition; mean (standard deviation).

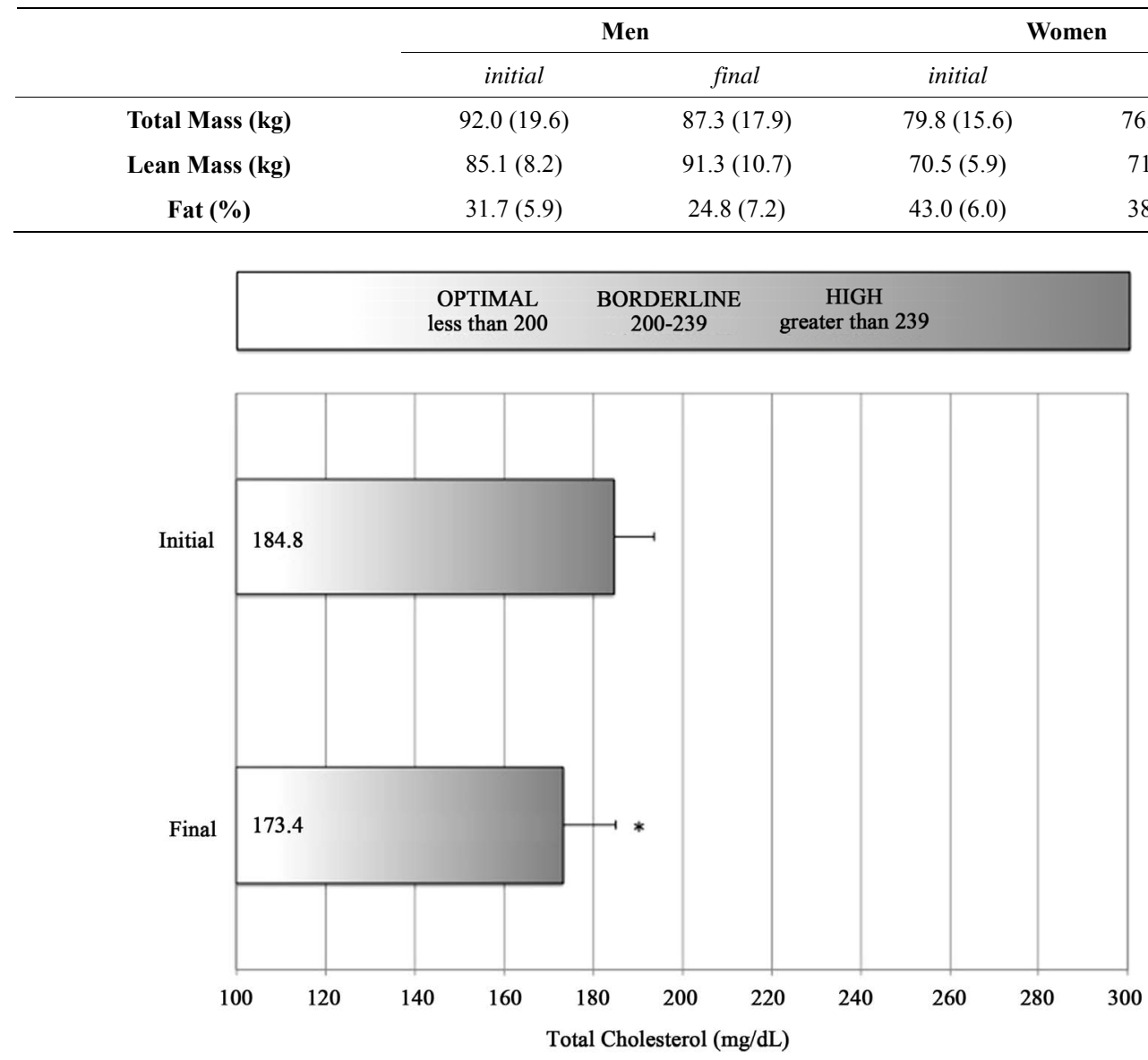

(a)

$\begin{array}{cccccc}\text { OPTIMAL } & \text { NORMAL } & \text { LOW } & \text { BORDERLINE } & \text { HIGH } & \text { EXTREME } \\ \text { less than 70 } & 70-99 & 100-129 & 130-159 & 160-189 & \text { greater than 19 }\end{array}$

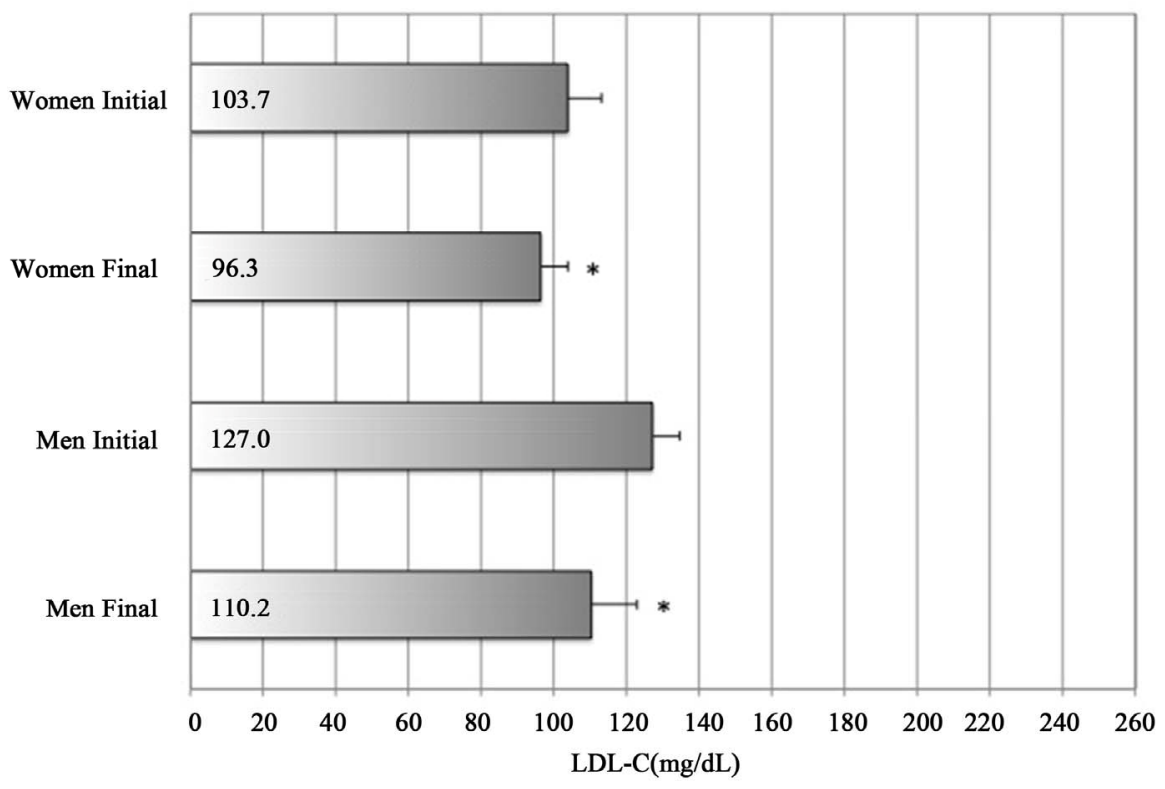

(b) 

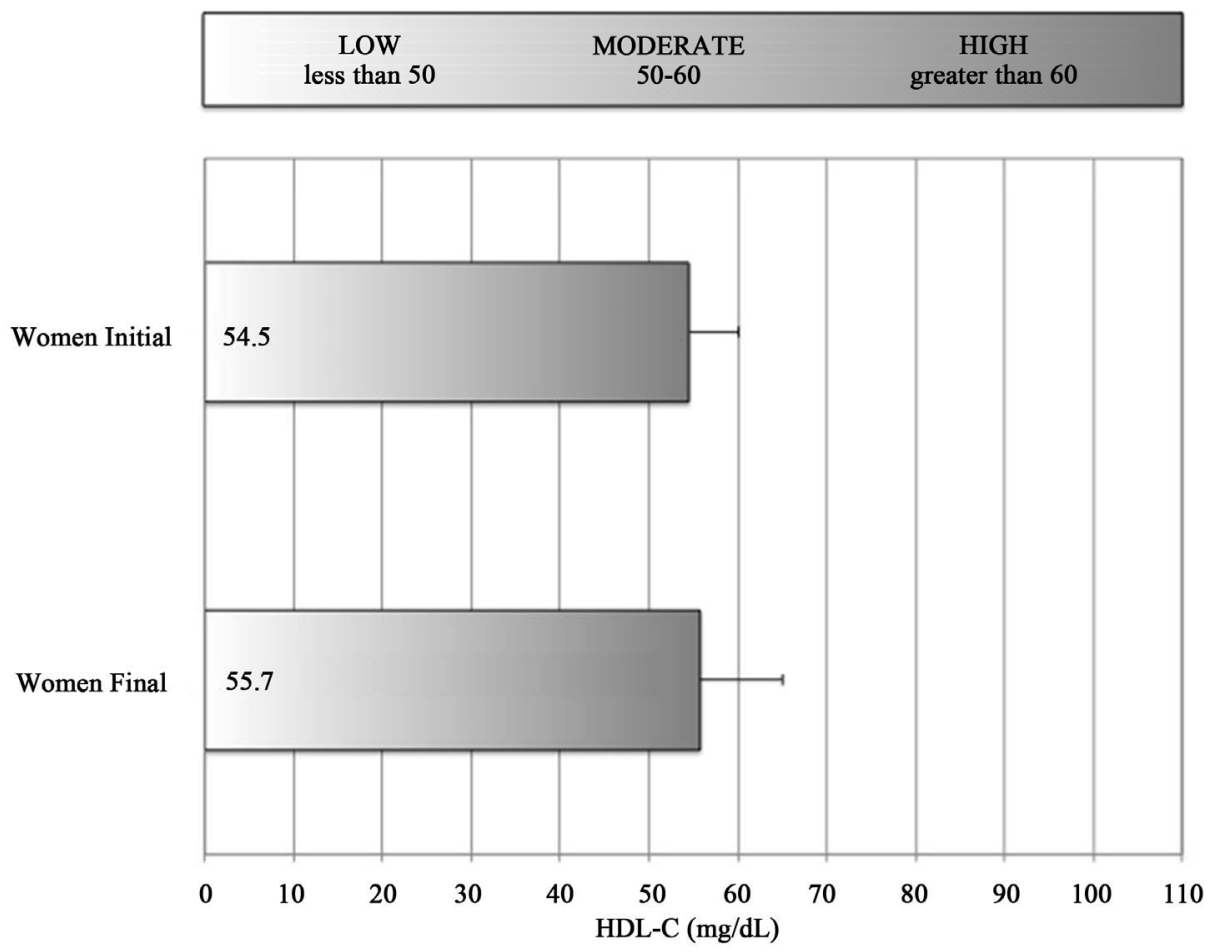

(c)

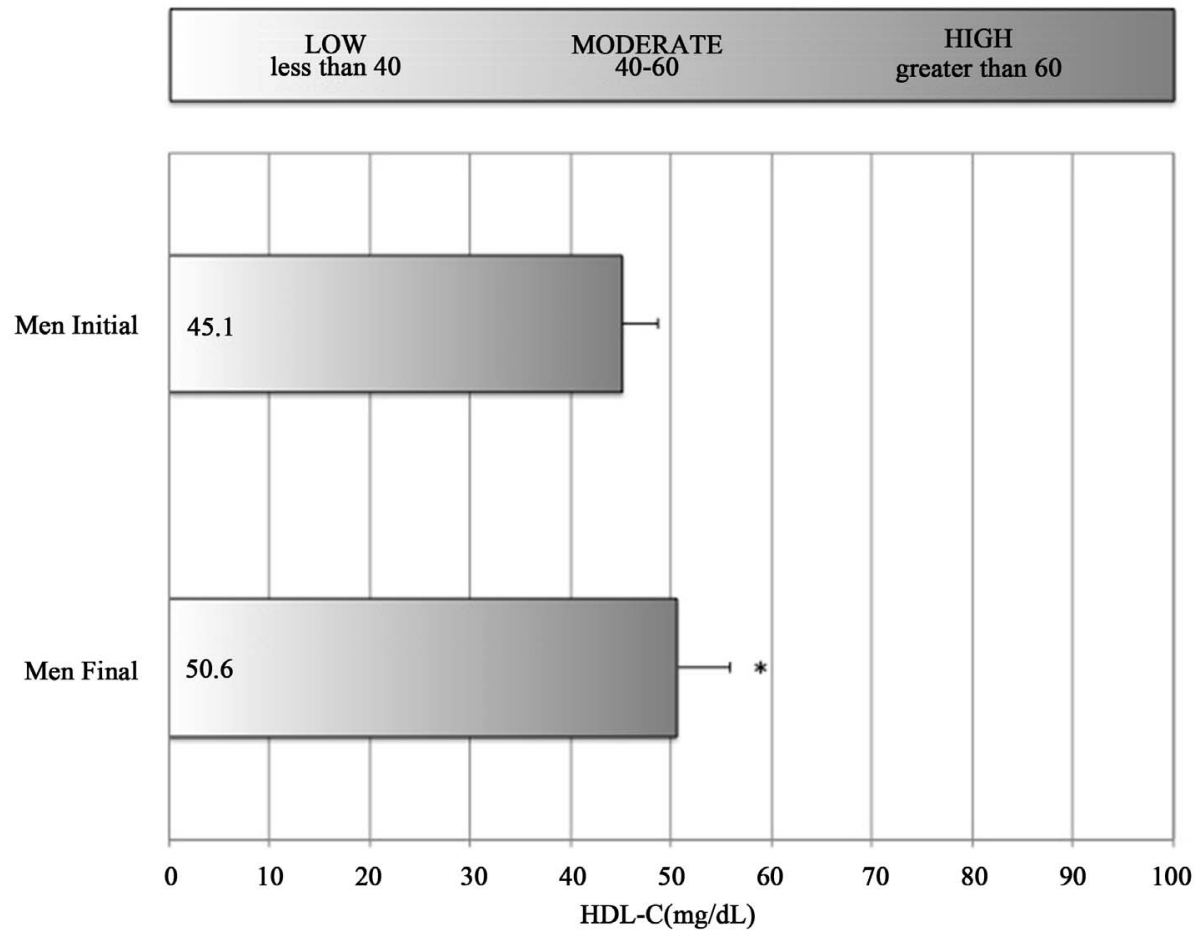

(d)

Figure 2. Mean \pm standard deviation for the initial and final measurements of total cholesterol concentration for women and men combined (a), low-density lipoprotein concentration for women and men (b), high-density lipoprotein concentration for women (c), and high-density lipoprotein concentration for men $(d)$. The asterisk indicates a significant difference $(p<0.05)$ between the initial and final measurements. The bar at the top of the figure illustrates the category and range for each variable. Note: Women and men have a different target range and are therefore represented in separate graphs. 


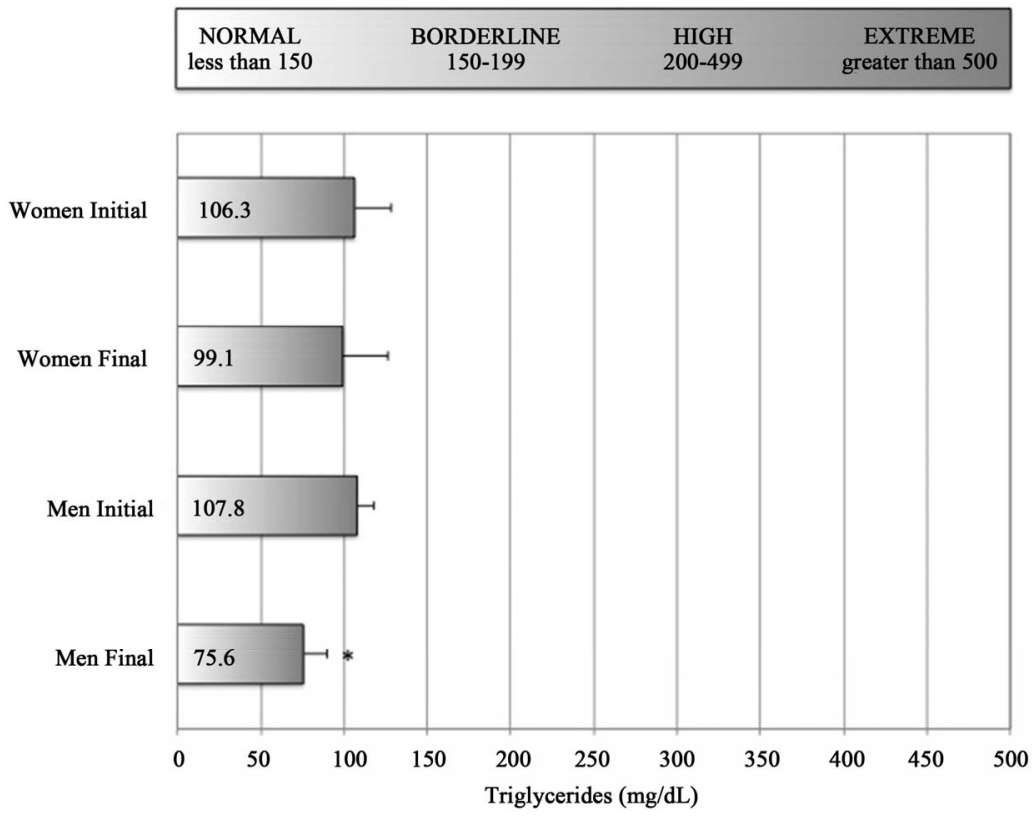

Figure 3. Mean \pm standard deviation for the initial and final measurements of triglycerides for men and women combined. The asterisk indicates a significant difference $(p<0.05)$ between the initial and final measurements. The bar at the top of the figure illustrates the category and range for each variable.

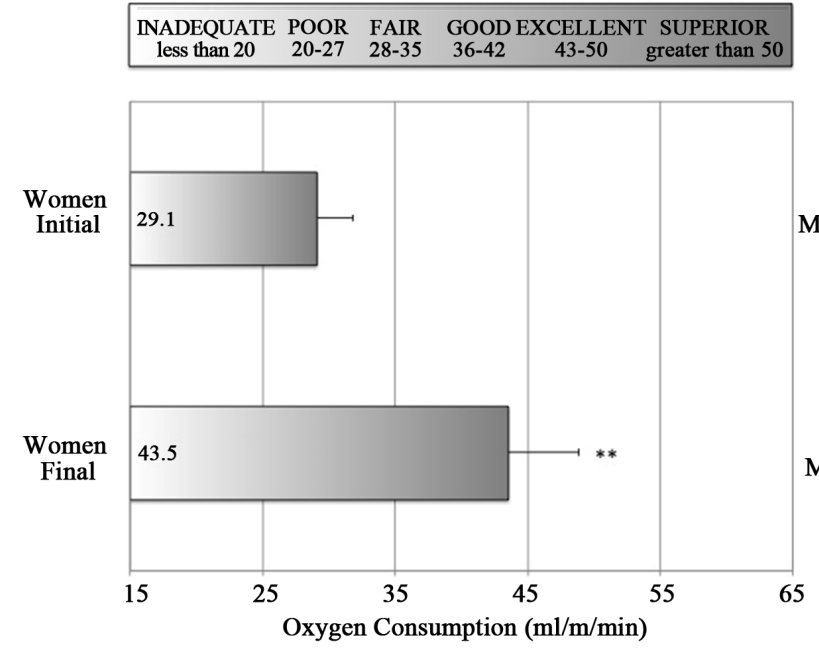

(a)

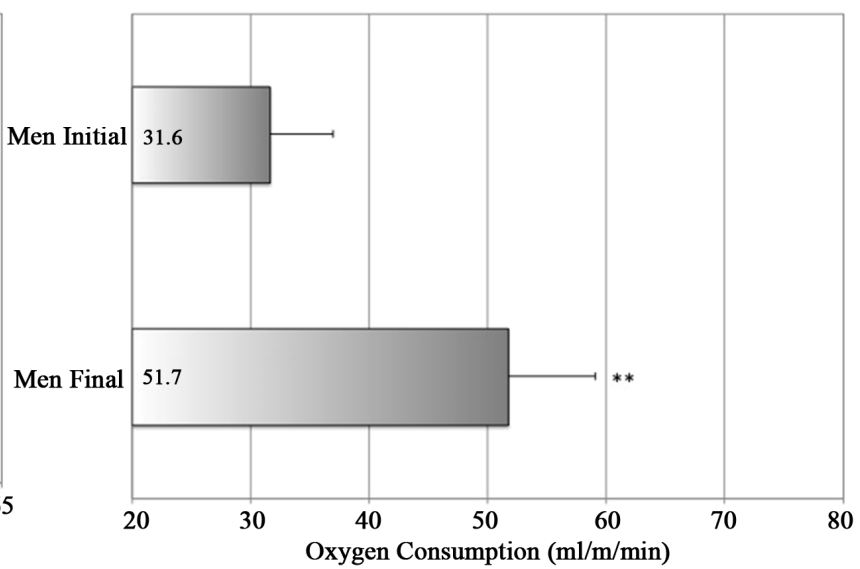

(b)

Figure 4. Mean \pm standard deviation for the initial and final measurements of oxygen consumption for women (a) and men (b). The double asterisk indicates a significant difference $(\mathrm{p}<0.01)$ between the initial and final measurements. The bar at the top of the figure illustrates the category and range for each variable. Note: Women and men have a different target range and are therefore represented in separate graphs.

insurers, and quality-of-care organizations whose goal is to implement innovative and practical strategies to reduce obesity, recommend a $5 \%$ to $10 \%$ sustained reduction in body mass as the appropriate measure of success for effective interventions [11]. The mean body mass for the men in the study was reduced by $5.1 \%$ from baseline and on an individual basis 6 men and 5 women had reductions of at least $5 \%$.
The protocol of this study differed from previous methods, as the participants in the current program were able to choose the type of cardiorespiratory class as well as their weekly schedule. The participants in the referenced studies were limited to a single cardiorespiratory exercise and a single strength training routine on specific days of the week for the duration of the respective study [5-7]. Also, they completed their workouts in a labora- 


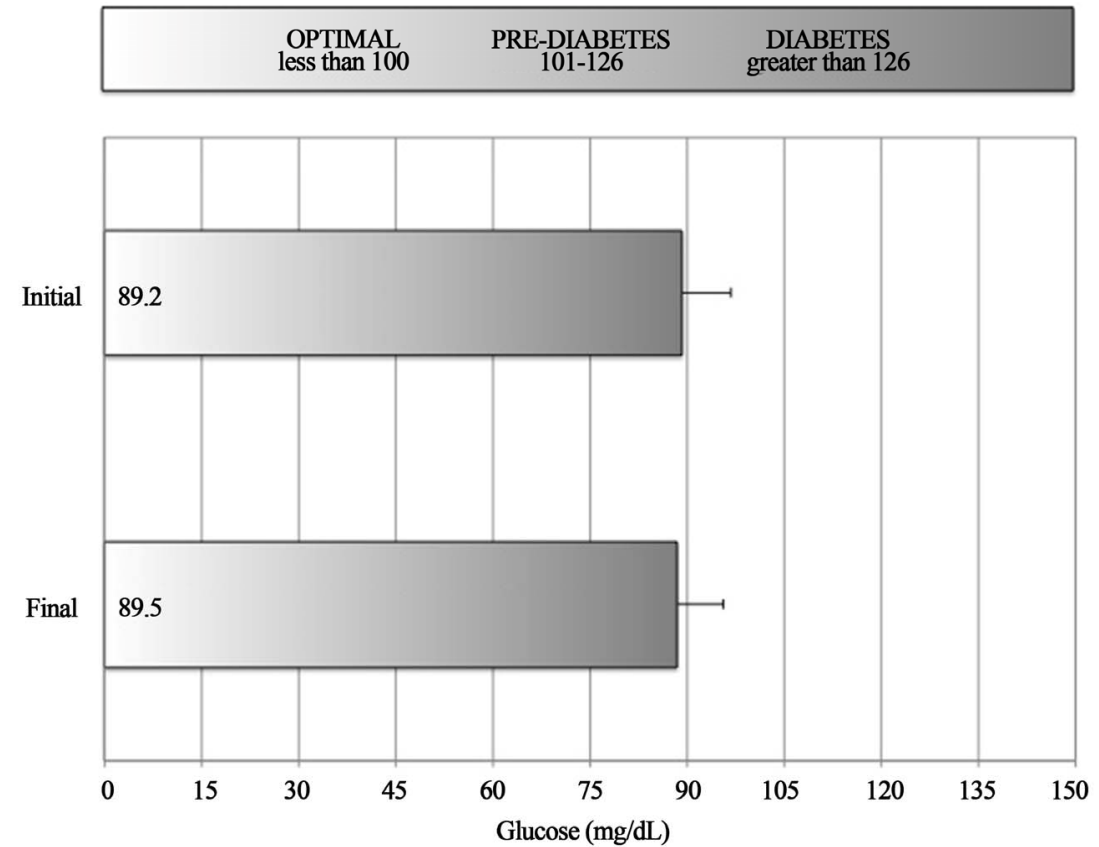

Figure 5. Mean \pm standard deviation for the initial and final measurements of glucose for men and women combined. The bar at the top of the figure illustrates the category and range for each variable.

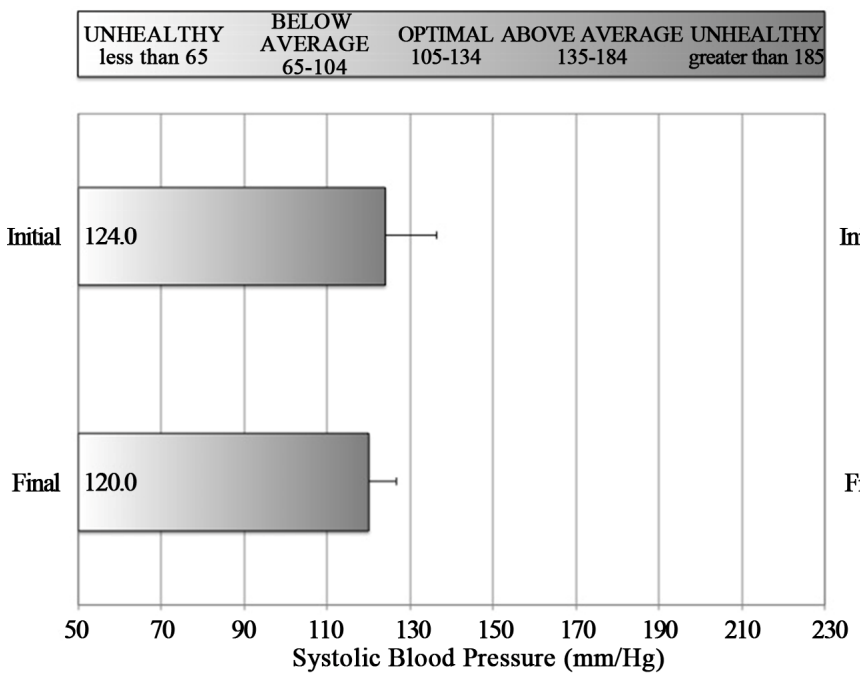

(a)

\begin{tabular}{|ccccc|}
\hline UNHEALTHY & BELOW & OPTIMAL & ABOVE AVERAGE & UNHEALTHY \\
less than 45 & AERAGE & OB-105 & greater than 105 \\
\hline
\end{tabular}

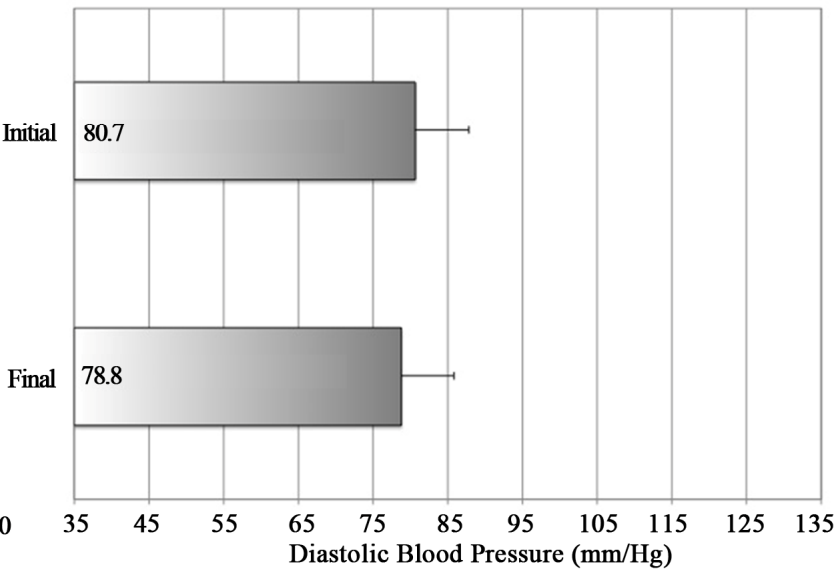

(b)

Figure 6. Mean \pm standard deviation for the initial and final measurements of systolic blood pressure (a) and diastolic blood pressure (b) for men and women combined. The bar at the top of the figure illustrates the category and range for each variable.

tory setting and were continually monitored by a research assistant. The participants in the present study completed their workouts in the typical fitness class setting at a local gym which applies to another STOP Obesity Alliance goal of obtaining actionable research with methods that are applicable in actual practice found in everyday life [11].

Overall, even with the independent schedule and unsupervised sessions, our data are comparable to past re- ports and even surpass numerous studies referenced in The American Heart Association (AHA) exercise intervention meta-analysis. For example, the AHA states that the average increase in HDL-C concentration is $4.6 \%$ from baseline values [12]. The men in the present group fitness program had a statistically significant increase in HDL-C concentration of $12.2 \%$ from the baseline value. To add, the average decrease for triglyceride concentration is $3.7 \%$ while the decrease with the current protocol 
was $29.9 \%$ for the men. The greater differences from the AHA values may be due to the higher exercise intensity from the group fitness classes. The participants in the present study wore heart rate monitors with a downloadable data component during each class and in the future we will expand upon the topic of intensity with a full analysis of the exercise zones.

The primary limitation of this study was the absence of a control group. Due to our promising results, we intend to expand upon this pilot study and compare this protocol to similar fitness regimens. We also plan to include dietary journals to track calorie consumption and nutritional intake for the duration of the program. Participants were asked not to alter their daily diet habits but we do not have any formal data to demonstrate that diet was not a significant factor. Despite these limitations, our data indicate that a 30 -week multi-modal group fitness program can satisfy the physical activity recommendations while minimizing attrition and maximizing health benefits.

\section{ACKNOWLEDGEMENTS}

We thank The Pennsylvania State University Social Science Research Institute for the funding to complete the study and The Athletic Clubs of State College for providing complimentary memberships. We also want to recognize the dedicated participants, their work created these impressive results. Finally, the study would not be possible without talented Clinical Research Center staff or the elite North Club instructor team.

\section{REFERENCES}

[1] 2008 Physical Activity Guidelines for Americans (2008) Health and human services. Washington DC.

[2] Centers for Disease Control and Prevention (2012) Be one in a million this American heart month.
[3] Surgeon General (1999) Physical Activity and health promotion. Atlanta.

[4] (2009) ACSM's guidelines for exercise testing and prescription. Lippincott Williams \& Wilkins, Philadelphia.

[5] Park, S.K., Park, J.H., Kwon, Y.C., Kim, H.S., Yoon, M.S. and Park, H.T. (2003) The effect of combined aerobic and resistance exercise training on abdominal fat in obese middle-aged women. Journal of Physiological Anthropology and Applied Human Science, 22, 129-135. doi:10.2114/jpa.22.129

[6] Libardi, C.A., De Souza, G.V., Cavaglieri, C.R., Madruga, V.A. and Chacon-Mikahil, M.P. (2012) Effect of resistance, endurance, and concurrent training on TNF-alpha, IL-6, and CRP. Medicine \& Science in Sports \& Exercise, 44, 50-56. doi:10.1249/MSS.0b013e318229d2e9

[7] McCarthy, C.J., Mills, P.M., Pullen, R., Roberts, C., Silman, A. and Oldham, J.A. (2004) Supplementing a home exercise programme with a class-based exercise programme is more effective than home exercise alone in the treatment of knee osteoarthritis. Rheumatology (Oxford), 43, 880-886. doi:10.1093/rheumatology/keh188

[8] Nielsen, A.C. (2007) National readership survey and panorama. Auckland.

[9] (2010) International health, IHRSA's member census.

[10] Donnelly, J.E., Blair, S.N., Jakicic, J.M., Manore, M.M., Rankin, J.W. and Smith, B.K. (2009) American college of sports medicine position stand. Appropriate physical activity intervention strategies for weight loss and prevention of weight regain for adults. Medicine \& Science in Sports \& Exercise, 41, 459-471. doi:10.1249/MSS.0b013e3181949333

[11] (2012) Strategies to overcome and prevent obesity alliance: Policy recommendations. D.o.H. Policy, Washington DC.

[12] Thompson, P.D. (2003) Exercise and physical activity in the prevention and treatment of atherosclerotic cardiovascular disease. Arteriosclerosis, Thrombosis, and Vascular Biology, 23, 1319-1321. doi:10.1161/01.ATV.0000087143.33998.F2 